\title{
A Study on Real-time Cooperative Ship Design System
}

\author{
Song Du \\ Ship CAD Engineering Center, Dalian University of \\ Technology, Dalian, Liaoning, China \\ dusong_2002@163.com
}

\begin{abstract}
Cooperative working mode is among the important ways to improve the efficiency and quality of ship design. This paper conducts a research on the real-time cooperative working mode for ship design, which is developed by means of compound sharing mode and in which the application of such two modes of transmission as strong link and weak link makes the system real-time and effective. Based on the general design platform, the remotely real-time cooperative ship design is accomplished. Thanks to this mode of design, the ship design cycle could be effectively shortened and correspondingly the cost of design is cut down.
\end{abstract}

Keywords-Cooperative design;ship design; XML; SVG; realtime cooperative

\section{INTRODUCTION}

During the process of ship design, it's inevitable that technicians with different majors and various identities may simultaneously have discussions about the same design drawing and make some corrections, as is shown in Figure1. Usually, these technicians are in different places, which mean the discussions about the design results may be conducted mostly via internet or e-mail for transmitting the design drawings and other relevant information, let alone the necessity of discussions in the same place at times. All these factors make the cost of design increased and the cycle of design prolonged. For the sake of the complexity of ship design, the Tribon M3 platform ${ }^{[1]}$, currently applied in the process of ship design and production, to some extent, may allow the cooperative design of the ship design, electrical system, mechanical system and cargo system, while there is no excellent software system to support the cooperative design work in the stage for preliminary ship design as well as in the stage for detailed design.

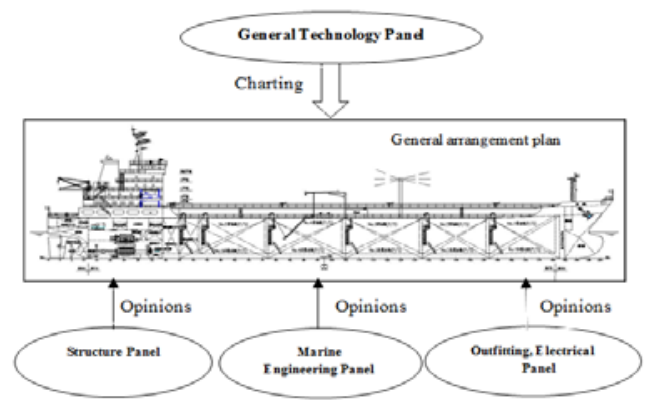

Figure 1. the Charting and Modification of a Ship’s General Arrangement Plan

\author{
Yan Lin \\ Ship CAD Engineering Center, Dalian University of \\ Technology, Dalian, Liaoning, China \\ linyanly@dlut.edu.cn
}

In this paper, the general engineering design software commonly used in ship design is taken as the main interactive platform, the application of XML technology; Object ARX and socket technology make the remotely distributed design system achievable that allows many cooperative designers to cooperatively work on the same design drawing. In addition, the real-time Web release of the design drawing is accessible with the aid of SVG technology. The XML file format being the data storage format and the means of the strong link and the weak link in a ring structure ensure the real time and effectiveness of data transmission in the running process of the system.

\section{THE FRAMEWORK OF THE REAL-TIME COOPERATIVE SHIP DESIGN SYSTEM}

\section{A. System Requirements}

The cooperative ship design is a cooperative process in which the main body, namely, the design organizer, makes connection with and control of the design participators and work together for designing a product. As is shown in Figure2, the ship designers are the core of the whole process of cooperative design. Their design concept is delivered by CAD software and interface program to the ship data model. Thus, the data in the DM is modified. Simultaneously, the process of modification is in turn transmitted to the middle module by CAD network interface. Other users of the cooperative design system could witness all the real-time modification which makes the modifications and intentions perceivable and open to every designer.

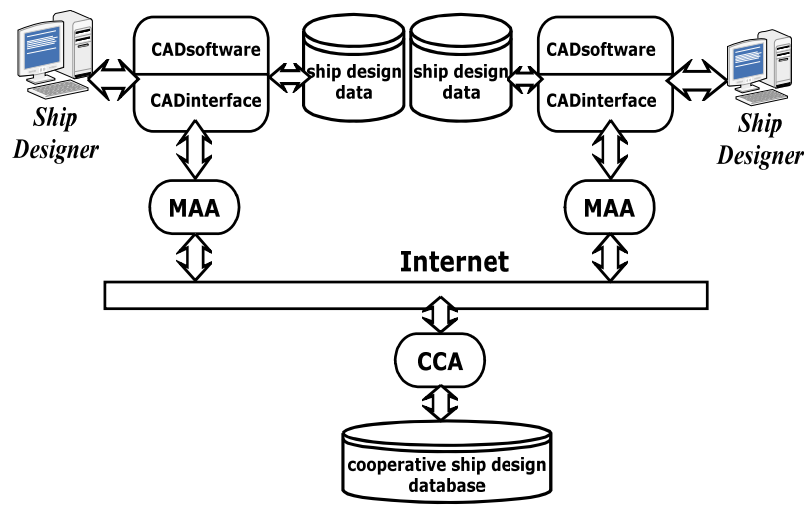

Figure 2. the Framework for Real-time Cooperative Ship Design System 
Apart from the function of design, the real-time cooperative ship design system shall meet the following requirements as well:

a. Team work. Cooperation is an important guarantee for successfully completing the ship design. The rational permission assignments among the designers and a favorable cooperation mechanism will be helpful for the improvement of the design quality and efficiency.

b. Cooperative awareness. In the process of real-time cooperation of ship design, the real-time communication plans accessible to various medias could be provided and a friendly interactive environment should be provided to the ship designers for perceiving others' design activities.

c. The sharing and management of the design data. The real-time cooperative design system shall manage the information concerning the design data and design process as well as provide unified data storage format which must be consistent, complete and continuous.

d. Dynamic and opening. The various parts of the cooperative design environment are required to be closely integrated, the interactive operation transparent, and the system platform relatively open.

\section{B. Network Organizational Structure}

The structural form of the logic network of the system is a compound sharing mode which absorbs the merits of both the centralized structure and the distributed structure ${ }^{[2,3]}$. The compound sharing mode with a better openness and versatility not only ensure a better consistency of data, but also ease the network burden and make the interconnection of the heterogeneous platforms more convenient. The network structure model of the system is shown in Figure3.

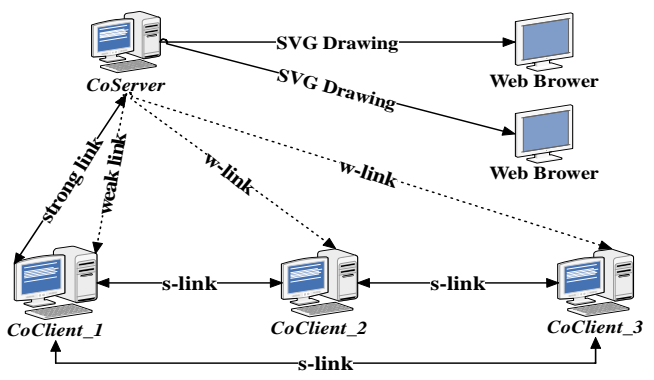

Figure 3. Network Structure

The organizer, as the chairman, is in charge of establishing the application service(Co Server) for the cooperative working group, while the product designers or experts with the remote ship design software platform start up the server Co-Client, firstly making a register on CoServer, then making connection as soon as the registration is approved. The link mode of Co-Client adopts a ring structure for the participation in the discussions and modifications of the design drawings. To those experts without the ship design engineering software, by logging on their own computers, the design drawing with a SVG format released by Co-Server would be available for a remotely real-time browsing. They could get to know the modifying situation of the product in the cooperative process as well as propose some opinions.

\section{The Cooperative Design System Module}

The cooperative design system mainly comprises of the Database Module, the Cooperative Management and Control Module, the Event Reactor, the Encoder, the Decoder, the Communication Module, the Data Check Module, the Web Release Module and CAD Display Module. Among them, the cooperative management and control module and the Web release module fulfill their function on the Chairman server. What is shown in Figure4 is the structure of the system's main modules for the communication between CoServer and Co-Client.

The data recorded in the database contains graphic data, users' information and operation records. With the help of the Event Reactor, The system conducts supervision and management of any design operations on the design drawing. Besides, the design data and the activities involved in the cooperative working group are stored in XML format. The encoder is to encode the graphic data and information data which will be generated into DOM tree in XML format by the use of Microsoft MSXML analyzer, facilitating the data management and the generation of the graphic files with a SVG format. With the assistance of Socket and the Event Reactor offered by Object ARX, the participators of the cooperative design in the internet environment could monitor the activities in the cooperative working group and make reactions.

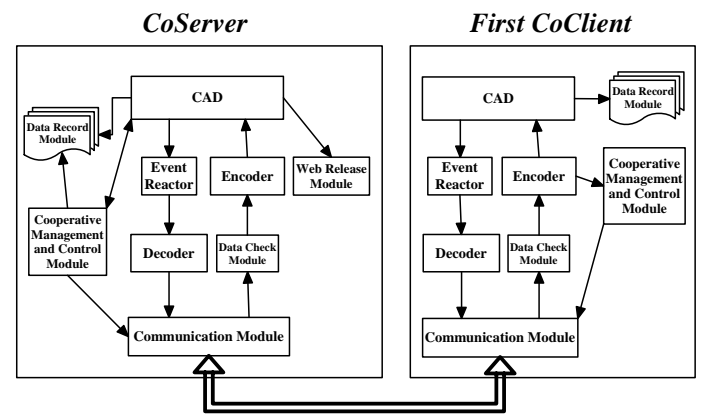

Figure 4. the System Communication Mechanism

\section{The synchronization Strategy of the Real-time Cooperative Design System}

The ship design information is re-encoded in a lighter encoding mode in order to realize the real-time cooperation among the design platforms. In the process of the cooperative ship design, the data is stored in XML format. What's more, the application of strong link and weak link in the ring structure keeps the data transmission in the running process of the system real-time and effective.

The Co-Server on the Chairman side, possessing the supreme authority of manipulating the design drawing, may authorize Co-Client to modify the design drawing. At a specific moment, there is only one designer who could have the right to modify the data. Namely, only this authorized Co-Client or Co-Server could send design data to the web, 
other modifications of the design from other designers failing to be perceived. The Co-Server socket is composed of four threading: 1. monitoring the users' application for permission; 2. monitoring the transmitted graphic data; 3 . sending data by weak link to all the designers; 4 . Send data by strong link to First Co-Client.

\section{E. the data transmission format}

Performing the real-time cooperation raises a requirement that the system needs to define its CAD Protocol, including: CAD Data Protocol for CAD graphic data communication among cooperative users; Control Protocol for the protocol support of the consistency maintenance and concurrency control; Macro Protocol for the control of the cooperative design under multi-input cooperation; Notice Protocol for the locking of the design discussions and design objects, etc.; and Task Protocol for the control of task query, the logging on , application and logging off of the users, etc..

\section{F. The Web Release}

By virtue of the interactive web pages on the internet, Co-Server dynamically sets up the design drawing in the SVG format, offering the real-time updated design drawing to the users which makes the visualized sharing based on Web realizable.

\section{The Key Technology for the ReALization of THE SYSTEM}

\section{A. The Data Transmission}

Currently, most network environment could not wholly support IP multi-casting. In order to solve the problem of limited amount of broadband and guarantee the high realtime and reliability of cooperative design, a compound data sharing mode blending the two kinds of data transmission rules---the strong link and weak link has been adopted.

Strong link(S-Link): all the graphic data and control information generated in the design process are sent and received between two platforms. The graphic data mentioned herein contains the original graphic data and the real-time modified graphic data. The strong link makes the real-time interaction the most active.

Weak link (W-Link): a small amount of data may be transmitted between two platforms. The weak link merely permit sending and receiving a small amount of control information and the header of the graphic data to ensure the management of the cooperative tasks and the maintenance of the data consistency.

\section{B. Event Reactor}

For the purpose of establishing the WYSIWIS environment under the CSCD system, when the graphic objects have changes, the modified design information is selectively sent to all the designers. Through the ACD Database Reactor derived from Object ARX, all the events (e.g. Entity-Adding, deletion and modification), generated in the graphic database of the ship design engineering software, are monitored in a real-time pattern to acquire the modified information of the entities in the cooperative process.
Afterwards, the information will be encoded in accordance with the type of the event and correspondingly the graphic files will be modified, recorded and sent to the next remote node by the means of Uni-cast. The monitoring threading at the receiving end will be started up to receive the real-time data and information, which then will be decoded after passing the Data Check Module and be added to the log. The current graphic database will be revised and refreshed for display. The event is synchronically transmitted.

\section{The Management of Authority}

Through the weak link, Co-Server conducts the consistency check and concurrence control of the data objects in the working group. The operations among the cooperative members are under control by defining the CO_SHARELOCK and the CO_EXCLUDELOCK in the system. The control of the modification of the shared objects and the access to handles makes only one user authorized at a time for modifying the shared objects, which in turn ensures the consistency of the data.

\section{Data Management}

The data storage mode adopt semantic sharing pattern and distributed storage pattern. Therefore, it is of necessity to keep the consistency of the model data among designers. The organization of the model data should observe some rules. The XML file format is adopted in the system to record such cooperative information as graphics, modification time, manipulator concerning the ship design drawing and the information of all the cooperative members. This way of data management features completeness, augment-ability and reproducibility as well as a compact structure and a lower system cost.

\section{E. SVG Technology}

SVG (Scalable Vector Graphics), as a vector graphic standard developed by W3C, is an application of XML (Extensible Markup Language) $\left[{ }^{4]}\right.$. Mainly for network application, SVG is flexible, extensible, better interactive, reproducible and elastic. As vector graphics, SVG graphics could be magnified deliberately without distortion, which is significant for the description of the ship structure arrangement. What is shown in Figure5 is a typical design drawing of a ship's bulkhead structure in SVG expression.

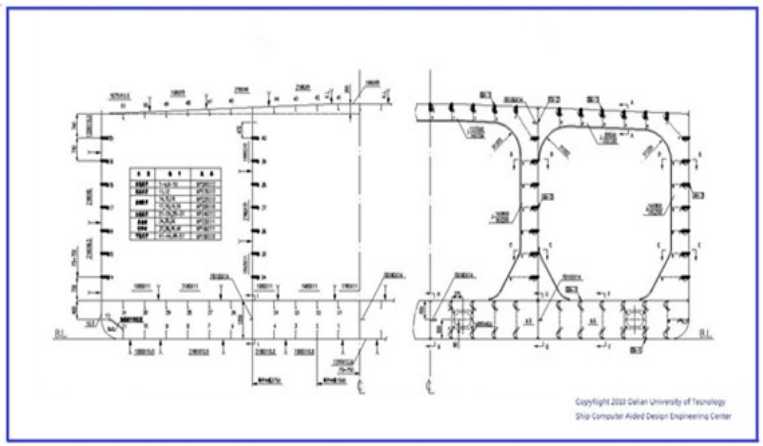

Figure 5. SVG expression of typical transverse bulkhead structure 


\section{PRototype System}

As is shown in Figure 6, here is a case of a cooperative ship design. In the case study, the cooperative design is conducted on a ship's general arrangement plan. The participating members, consisting of the General Technology Panel, the Structure Panel and the Marine Engineering Panel in the same local area network (LAN) and the ship owner in the WAN, participate in the cooperative design at various terminals. The General Technology Panel, as the chairman, launches the invitation for the real-time cooperative design. Also, As the Server, the General Technology Panel owns the supreme authority of the design drawing; the Structure Panel participates in the real-time cooperative modification of the general arrangement design plan. The members of the Structure Panel shares the same authority with their counterparts in the General Technology Panel in terms of the real-time modification of the general arrangement plan; the members of the Marine Engineering Panel could receive the real-time modifications of the general arrangement plan from the General Technology Panel and the Structure Panel via the ship design platform as well as propose some opinions for modifications in the form of voice text; while the ship owner may use Web browser to update the real-time general arrangement plan and obtain the vector graphic design expressed in SVG as well as take part in the communication via e-mail or voice text.

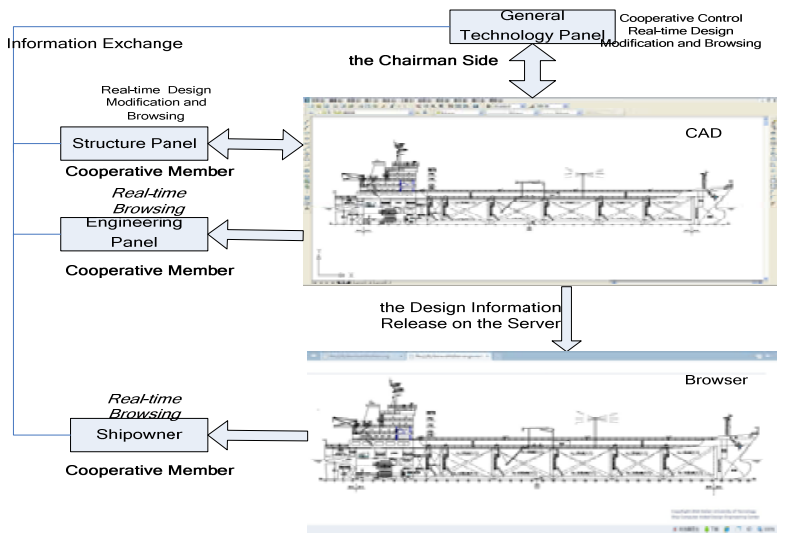

Figure 6. the Prototype System

\section{A. The Construction Process of Cooperative Design Working Panel}

The Chairman starts up Co-Server for accepting the application for participation while the remote client starts up Co-Client for applying for joining in the cooperative panel. As soon as the application is approved, the connection will be established. Then Co-Server chronologically initializes the client list to establish the brotherhood among the clients. Afterwards, Co-Server will establish the S-link with the first Co-Client and W-link with all Co-Clients for sending the Web users' information to every participator, which is stored in the Co-client and is accessible for discussions. After the initialized dated are sent, all the Co-Clients confirm the information and give feedback. The Chairman checks that the network has been established and begins to send graphic design data, which shows the working panel has been built. The construction process of the working panel is shown in Figure 7.

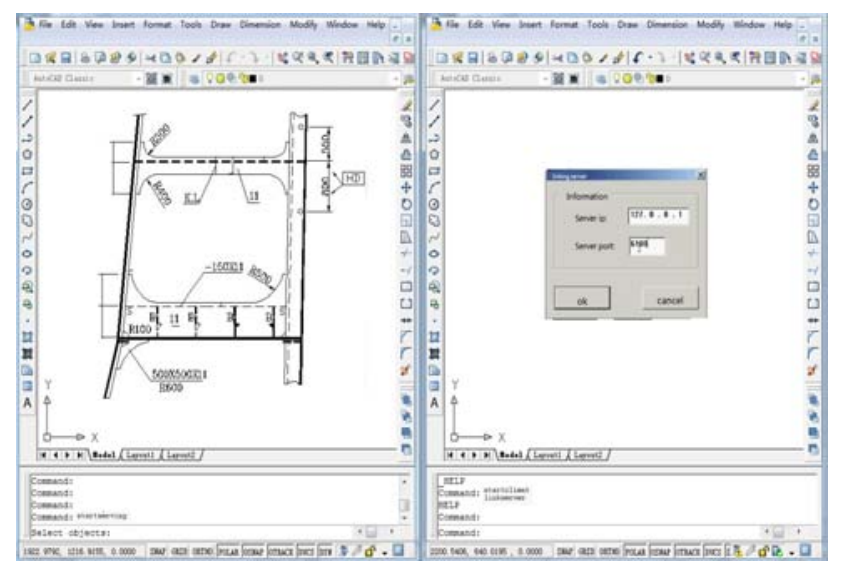

Figure 7. the Establishment of Cooperative Panel

\section{B. The Initialization of the Design Information of the Working Panel}

Co-Server sends the control information of the design drawing to all the participators and all the current graphic data involved to the first Co-Client. The first Co-Client receives the data and simultaneously sends it to other CoClients nodes. After receiving the end mark, the Co-Client starts to check whether the received data and the headline date are consistent. As soon as the check proves errorless, the Co-Client decodes the data and put it CAD working area for display. Meanwhile, the data and Uniform ID will be stored in the XML file. After the completion of the date-sending, by use of the order---zoom all in every Co-Client, all the interfaces of the members become identical at this moment, which signifies the completion of the initialization of the graphic data. What is shown in Figure 8 is the synchronization of the ship design data.

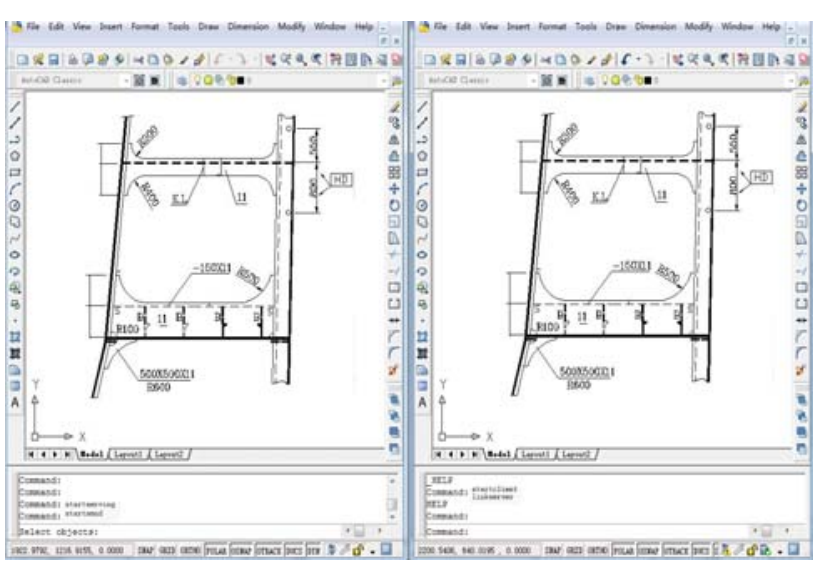

Figure 8. the Initialization of The Graphic Data 


\section{Sending the Real-time Modified Graphic Data}

Every cooperative user may enjoy this function. The change of the graphic data could be perceived via Reactor Module and sent selectively. There are two sending modes: one mode is real-time data transmission, which makes every single modification accessible simultaneously on other cooperative servers; the other one is incremental data transmission, by which the data controller firstly makes partial modifications and then sends the modified data in batches to the cooperative members. This process is shown in Figure 9.

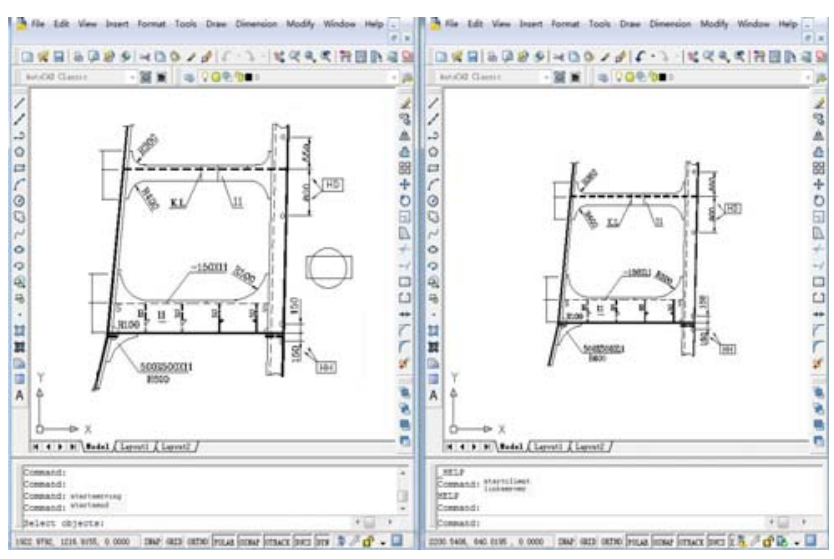

Figure 9. Real-time Cooperation

\section{SUMMARY}

With the system platform featuring adopting the B/S and $\mathrm{C} / \mathrm{S}$ compound system in the Web environment, the real-time cooperative ship design system makes achievable the information sharing and concurrence control in real-time cooperative design based on the general design platform. The real-time system framework in this paper could be applied on various ship design engineering platforms for the real-time interactive operation in different places could be accomplished by re-developing the system. This paper places weight on such key technology as the system structure, multi-user interface, concurrence control, consistency check, access control and record management on the basis of the real-time cooperative ship design platform. However, this paper does not touch on other key technology like version control, task planning and conflict elimination, which needs for further research in the future.

\section{REFERENCES}

[1] Qiao Shan Ying Wen-ye He Gang. Application of Digitalization Design for Ship [J].Chinese Journal of Ship Research, 2007, 4:20-25

[2] Francis Paling,Nicola Senin and David Wallace.Distribution modeling and evaluation of product design problems [J].ComputerAided Design,2008,30(6): 411-423.

[3] Hisup Park and Mark R.Cutkosky. Framework for modeling dependencies in collaborative engineering processes [J].Research in Engineering Design, 2009, 11:84-102.

[4] Scalable Vector Graphics (SVG) 1.1 Specification.W3C 2003 http://www.W3.org 